MIRANDA, N.O.; MEDEIROS, J.F.; NASCIMENTO, I.B.; ALVES, L.P. Produtividade e qualidade de frutos de melão em resposta à cobertura do solo com plástico preto e ao preparo do solo. Horticultura Brasileira, Brasília, v. 21, n. 3, p. 490-493, julho-setembro 2003.

\title{
Produtividade e qualidade de frutos de melão em resposta à cobertura do solo com plástico preto e ao preparo do solo
}

\author{
Neyton O. Miranda; José F. Medeiros; Iarajane B. Nascimento; Leonardo P. Alves
}

ESAM, Depto. Engenharia Agrícola, C. Postal 137, 59625-900 Mossoró-RN. E-mail: neyton@esam.br

\begin{abstract}
RESUMO
Determinou-se o efeito da intensidade do preparo e da cobertura do solo com plástico preto sobre a produção e qualidade de frutos de melão irrigado por gotejamento. $\mathrm{O}$ experimento foi conduzido em Mossoró, no delineamento blocos ao acaso em esquema fatorial com parcela subdividida, com três repetições. Foram avaliados o tipo de preparo do solo (área total ou em faixas) e profundidade de preparo $(20 ; 30 ; 40$ e $50 \mathrm{~cm})$, com parcelas divididas em com ou sem cobertura do solo. Os frutos foram classificados em tipo exportação, mercado interno e refugo para determinar peso de frutos de cada tipo, número total de frutos e seu peso médio. Foram determinados a firmeza de polpa, o teor de sólidos solúveis totais (SST), espessura da polpa e formato do fruto. A qualidade dos frutos de melão foi reduzida pela cobertura do solo apenas em termos de firmeza de polpa. O preparo do solo em faixas não influenciou as características estudadas. Maiores profundidades de preparo aumentaram a produção de frutos tipo exportação, entretanto diminuíram a produção para o mercado interno.
\end{abstract}

Palavras-chave: Cucumis melo L., mecanização agrícola, sólidos solúveis totais.

\begin{abstract}
Yield and quality of melon fruits in response to plastic mulch and soil tillage

The effect of black polyethylene mulch and soil tillage intensity was determined on yield and quality of drip irrigated melon. The field trial was carried out in Mossoró, Brazil. The experimental design was randomized complete block in a factorial scheme with three replications. Two soil tillage methods were evaluated (strip tillage or tillage of the entire area) and tillage depth $(20 ; 30 ; 40$ and $50 \mathrm{~cm})$, with split plots with or without mulch. Fruits were classified in export type, internal market type and rejected, to determine total yield and the yield of each type, number of fruits and fruit mean weight. Pulp firmness, total soluble solids, pulp thickness and fruit shape were determined. Results showed a decrease in pulp firmness with black plastic mulch. Strip tillage did not influence any studied characteristic. Yield of both export type and internal market melons was influenced by tillage depth. While deep tillage increased yield of export type melons, it reduced internal market yield.
\end{abstract}

Keywords: Cucumis melo L., agricultural mechanization, total soluble solids.

(Recebido para publicação em 08 de maio de 2002 e aceito em 22 de abril de 2003)

$\mathrm{O}$ melão produzido no Nordeste brasileiro é competitivo no mercado externo. Porém, esta competitividade pode ser melhorada se forem adotadas alternativas para diminuir custos de produção. Para tanto, o preparo do solo e a irrigação são componentes importantes.

Operações de preparo são justificadas por retorno em produtividade maior que seu custo, ou melhoria nas condições físicas e biológicas do solo (Siemens et al., 1993), e pelo controle de plantas invasoras (Arndt \& Rose, 1966). Porém, o solo muitas vezes é preparado excessivamente por agricultores que preferem usar práticas e equipamentos tradicionais a correr riscos.

Existem alternativas ao preparo convencional que otimizam custos e eficiência, como operações combinadas, preparo em faixas e na profundidade necessária à produção, e variações em profundidade, largura preparada e intensidade de destorroamento, que simplificam, eliminam ou diminuem operações, economizam combustíveis, equipamentos, mão-de-obra e tempo, mantendo a produtividade (Hillel, 1982; Hunt, 1995; McKibben \& Whitaker, 1973; Unger \& McCalla,1981).

A economia de água, tempo, dinheiro e mão-de-obra justifica o preparo em faixas nos solos cultivados em linhas cuja compactação é definida e fácil de eliminar (Arndt \& Rose, 1966; Oschwald, 1973). Pequenas variações na profundidade do preparo causam grandes variações no tempo e na quantidade de combustível gastos, no desgaste e danos aos tratores e implementos (Unger \& McCalla, 1981). O preparo profundo $(40-45 \mathrm{~cm})$ chega a custar o dobro do médio $(35 \mathrm{~cm})$, quatro vezes mais que o normal $(25 \mathrm{~cm})$ e dez vezes mais que o raso $(15 \mathrm{~cm})$ (Hillel, 1982). Mesmo assim, a descompactação do solo e maior infiltração e armazenamento de água, devidos ao preparo profundo, podem aumentar a produtividade, principalmente em anos com deficiência hídrica (McKibben \& Whitaker, 1973; Trouse Jr. \& Humbert, 1959).

A cobertura do solo ('mulch') com plástico na cultura do melão, comum em vários países, teve introdução recente na região produtora do Rio Grande do Norte. Esta prática visa aumentar a produção e qualidade dos frutos, antecipar a colheita, reduzir a infestação de pragas e das doenças por estas disseminadas, e melhorar o controle de plantas invasoras. Também reduz danos e doenças nos frutos, pois diminui seu contato com o solo. A maior temperatura e menor amplitude térmica no solo coberto pode aumentar a taxa de desenvolvimento das plantas e antecipar a colheita. Em regiões semi-áridas a economia de água por redução nas perdas por evaporação é importante benefício da cobertura do solo. As condições de temperatura e umidade criadas no solo proporcionam economia de fertilizantes pela maior disponibilidade de nutrientes. No caso 
do nitrogênio, a cobertura favorece a nitrificação e reduz perdas por volatilização (Araújo, 2000; Ferreira, 2001; Ibarra et al., 2001).

Algumas vantagens observadas em pesquisas com melão, atribuídas ao uso de cobertura do solo com plástico, relacionam-se com o desenvolvimento vegetativo, número total de frutos, número de frutos comerciais, peso médio de frutos, produtividade total e comercial e teor de sólidos solúveis totais, logo após a colheita de melão amarelo (Silva, 2002); firmeza de polpa (Araújo, 2000); matéria seca, área foliar e rendimento de frutos (Ibarra et al., 2001); produção de frutos comerciais (Ferreira, 2001) e teor de sólidos solúveis totais, peso de frutos, número de frutos por hectare e produtividade total de melão cantaloupe (Maiero et al., 1987).

O objetivo desse trabalho foi determinar o efeito da cobertura do solo com plástico preto e o efeito da intensidade do preparo do solo sobre alguns componentes da produção e qualidade dos frutos de melão irrigado por gotejamento.

\section{MATERIAL E MÉTODOS}

O experimento foi implementado no município de Mossoró-RN, latitude $5^{\circ} 11^{\prime} \mathrm{S}$, longitude $37^{\circ} 20^{\prime} \mathrm{W}$ e altitude de $18 \mathrm{~m}$. O clima é muito quente, semi-árido, com chuvas predominando de fevereiro a maio. O solo da área é um LUVISSOLO CRÔMICO Pálico, profundo, poroso e bem drenado, com $\mathrm{pH}$ entre 7,1 e 7,3. Os teores médios de alguns elementos, na análise química do solo da camada entre 0 e $20 \mathrm{~cm}$ de profundidade, foram: $\mathrm{Ca}=8,4 \mathrm{cmol}_{\mathrm{c}} \mathrm{dm}^{-3}$; $\mathrm{Mg}=2,72 \mathrm{cmol}_{\mathrm{c}} \mathrm{dm}^{-3} ; \mathrm{K}=1,84 \mathrm{cmol}_{\mathrm{c}}$ $\mathrm{dm}^{-3} ; \mathrm{Na}=0,18 \mathrm{cmol}_{\mathrm{c}} \mathrm{dm}^{-3} ; \mathrm{e} \mathrm{P}=39 \mathrm{mg}$ $\mathrm{dm}^{-3}$. As frações granulométricas do solo da camada entre 0 e $30 \mathrm{~cm}$ de profundidade foram: areia grossa $=370 \mathrm{~g} \mathrm{~kg}^{-1}$; areia fina $=190 \mathrm{~g} \mathrm{~kg}^{-1}$; silte $=120 \mathrm{~g} \mathrm{~kg}^{-1}$; e argila $=320 \mathrm{~g} \mathrm{~kg}^{-1}$. A densidade do solo era de $1,58 \mathrm{Mg} \mathrm{m}^{-3}$ entre 0 e $15 \mathrm{~cm}$ de profundidade; $1,48 \mathrm{Mg} \mathrm{m}^{-3}$ entre 15

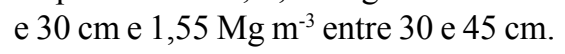

No preparo da área usou-se grade média com 28 discos de 0,59 m de diâmetro; escarificador de arrasto com rodas limitadoras de profundidade e has- tes com pontas retangulares de $0,08 \mathrm{~m}$ de largura; grade leve com 24 discos de 0,46 m de diâmetro; sulcamento; adubação no sulco $\left(8 \mathrm{~kg} \mathrm{ha}^{-1}\right.$ de $\mathrm{N}$ e $42 \mathrm{~kg}$ $\mathrm{ha}^{-1}$ de $\mathrm{P}_{2} \mathrm{O}_{5}$ ); construção de camalhões e semeadura manual. $\mathrm{O}$ trator utilizado, com potência de $106,6 \mathrm{~kW}$, possuía tração dianteira auxiliar e peso aproximado de $75500 \mathrm{~N}$.

O meloeiro amarelo, híbrido 'Gold Mine', foi semeado em 05/11/99 com espaçamento entre linhas de $2,0 \mathrm{~m}$, com duas plantas a cada $0,45 \mathrm{~m}$ espaçadas de $0,15 \mathrm{~m}$. A adubação foi feita via água de irrigação (68 kg ha-1 de N; $120 \mathrm{~kg} \mathrm{ha}^{-1}$ de $\mathrm{P}_{2} \mathrm{O}_{5}$ e $264 \mathrm{~kg} \mathrm{ha}^{-1}$ de $\mathrm{K}_{2} \mathrm{O}$ ). Os tratos culturais seguiram o sistema de produção da empresa. A colheita foi realizada nos dias 4, 11 e 17/01/00.

$\mathrm{Na}$ irrigação foram usados gotejadores espaçados de $0,60 \mathrm{~m}$ com vazão média de $2,3 \mathrm{~L} \mathrm{~h}^{-1}$ à pressão de $100 \mathrm{kPa}$. A evapotranspiração de referência foi calculada pelo método de Penman-Monteith com dados da Estação Climatológica da ESAM, situada a $12 \mathrm{~km}$ do local do experimento, e os coeficientes de cultivo recomendados por Allen et al. (1998). A lâmina de irrigação, até os 60 dias, foi de $222 \mathrm{~mm}$ nas parcelas descobertas e $200 \mathrm{~mm}$ nas cobertas.

Usou-se o delineamento experimental de blocos casualizados completos em esquema fatorial com parcela subdividida e três repetições. As parcelas receberam combinações dos fatores: tipo de preparo do solo (escarificação em área total ou faixas com 1,2 m de largura) e profundidades de escarificação (20; 30 ; 40 e $50 \mathrm{~cm}$ ), e foram divididas em descobertas e cobertas com filme de polietileno preto. As parcelas constavam de três linhas de melão, tendo sido colhidos 10,2 m de comprimento da linha central.

Os frutos foram classificados, por pessoa especializada, em tipo exportação, mercado interno e refugo, segundo critérios de tamanho, aspecto e injúrias mecânicas, da maneira como é feito na produção comercial de melão. Foram determinados: peso de frutos de cada tipo, número de frutos total e peso médio de frutos. Na primeira colheita tomaram-se quatro frutos por parcela para determinar: firmeza de polpa através da resistência à penetração usando penetrômetro com "plunger" de $8 \mathrm{~mm}$ de diâmetro; teor de sólidos solúveis totais (SST) usando refratômetro digital com compensação automática de temperatura; espessura de polpa e formato (relação entre diâmetro longitudinal e transversal do fruto).

Os dados obtidos foram submetidos à análise de variância e à comparação de médias pelo teste de Tukey a 5\% de probabilidade.

\section{RESULTADOS E DISCUSSÃO}

Não se observou efeito significativo, pelo teste $\mathrm{F}$ a $5 \%$ de probabilidade, dos fatores estudados sobre a espessura da polpa, formato do fruto e teor de sólidos solúveis totais nem suas interações (Tabela 1). Quanto à firmeza de polpa, apenas o fator cobertura do solo revelou efeito significativo, tendo o seu uso diminuído a firmeza dos frutos. Isto pode ser devido à maior retenção de umidade no solo coberto, como observado por Ferreira (2001). Porém, contraria Araújo (2000) que também observou maior umidade no solo coberto com plástico preto e menor firmeza de polpa no solo descoberto, que foi atribuída ao estresse hídrico. Os frutos com maior firmeza são mais resistentes às injúrias mecânicas durante o transporte e comercialização (Grangeiro, 1997). Os teores de sólidos solúveis totais estão, no geral, aquém dos valores esperados para exportação. Isto se deve, segundo Grangeiro (1997), ao fato de o método de determinação de SST em laboratório exigir a homogeneização da polpa, resultando em valores duas a três unidades menores do que o método empregado pela maioria das empresas produtoras que empregam algumas gotas do suco extraído de uma fatia de melão. A qualidade dos frutos de melão não foi influenciada pelo preparo do solo, sendo recomendável, segundo Hillel (1982), simplificar e diminuir as operações de preparo.

Dos componentes de produção de melão avaliados (Tabela 2) foi constatado que a produção de frutos do tipo refugo (PREF), o número total de frutos (NTOT) e o peso médio dos frutos (PMTOT) não foram influenciados pe- 
Tabela 1. Valores médios de características de qualidade de frutos de melão em resposta à cobertura do solo com plástico preto e ao tipo de preparo do solo. Mossoró, ESAM, 2000.

\begin{tabular}{lcccc}
\hline \multicolumn{1}{c}{ Fatores } & $\begin{array}{c}\text { Espessura de polpa } \\
(\mathbf{c m})\end{array}$ & Formato do fruto & $\begin{array}{c}\text { Firmeza de polpa } \\
(\mathbf{N})\end{array}$ & SST $^{1}$ ('Brix) \\
\hline Cobertura & & & & \\
Com & $3,84 \mathbf{a}^{*}$ & $1,21 \mathrm{a}$ & $35,02 \mathrm{~b}$ & $8,66 \mathrm{a}$ \\
Sem & $3,80 \mathrm{a}$ & $1,21 \mathrm{a}$ & $36,65 \mathrm{a}$ & $8,74 \mathrm{a}$ \\
\hline Preparo do solo & & & & \\
Total & $3,72 \mathrm{a}$ & $1,22 \mathrm{a}$ & $36,00 \mathrm{a}$ & $8,62 \mathrm{a}$ \\
Faixas & $3,91 \mathrm{a}$ & $1,21 \mathrm{a}$ & $35,68 \mathrm{a}$ & $8,77 \mathrm{a}$ \\
\hline Profundidade & & & & \\
$20 \mathrm{~cm}$ & $3,88 \mathrm{a}$ & $1,21 \mathrm{a}$ & $35,66 \mathrm{a}$ & $8,62 \mathrm{a}$ \\
$30 \mathrm{~cm}$ & $3,81 \mathrm{a}$ & $1,21 \mathrm{a}$ & $35,85 \mathrm{a}$ & $8,52 \mathrm{a}$ \\
$40 \mathrm{~cm}$ & $3,84 \mathrm{a}$ & $1,23 \mathrm{a}$ & $35,84 \mathrm{a}$ & $8,89 \mathrm{a}$ \\
$50 \mathrm{~cm}$ & $3,72 \mathrm{a}$ & $1,20 \mathrm{a}$ & $36,00 \mathrm{a}$ & $8,75 \mathrm{a}$ \\
\hline $\mathrm{C} . \mathrm{V} .(\%)$ & 13,18 & 1,66 & 3,88 & 8,57 \\
\hline
\end{tabular}

${ }^{1} \mathrm{SST}$ - sólidos solúveis totais. *Médias seguidas por letras iguais nas colunas não diferiram pelo teste $\mathrm{F}$ a $5 \%$ de probabilidade.

Tabela 2. Médias de alguns componentes de produção do meloeiro em resposta à cobertura do solo com plástico preto e ao preparo do solo. Mossoró, ESAM, 2000.

\begin{tabular}{lcccccc}
\hline \multicolumn{1}{c}{ Fatores } & $\begin{array}{c}\text { PNAC1 } \\
\left(\mathbf{k g ~ h a}^{-1}\right)\end{array}$ & $\begin{array}{c}\text { PEXP } \\
(\mathbf{k g ~ h a})\end{array}$ & $\begin{array}{c}\text { PREF } \\
\left(\mathbf{k g ~ h a}^{-1}\right)\end{array}$ & $\begin{array}{c}\text { PTOT } \\
\left(\mathbf{k g ~ h a}^{-1}\right)\end{array}$ & NTOT & $\begin{array}{c}\text { PMTOT } \\
(\mathbf{k g})\end{array}$ \\
\hline Cobertura & & & & & & \\
Com & $17282 a^{*}$ & $5794 \mathrm{a}$ & $6048 \mathrm{a}$ & $29124 \mathrm{a}$ & $13848 \mathrm{a}$ & $2,18 \mathrm{a}$ \\
Sem & $15047 \mathrm{a}$ & $6994 \mathrm{a}$ & $6372 \mathrm{a}$ & $28413 \mathrm{a}$ & $13399 \mathrm{a}$ & $2,08 \mathrm{a}$ \\
\hline Preparo & & & & & & \\
Total & $16152 \mathrm{a}$ & $6525 \mathrm{a}$ & $6522 \mathrm{a}$ & $28928 \mathrm{a}$ & $13440 \mathrm{a}$ & $2,10 \mathrm{a}$ \\
Faixas & $16177 \mathrm{a}$ & $6264 \mathrm{a}$ & $6168 \mathrm{a}$ & $28609 \mathrm{a}$ & $13807 \mathrm{a}$ & $2,16 \mathrm{a}$ \\
\hline Profundidade & & & & & & \\
$20 \mathrm{~cm}$ & $19185 \mathrm{~A}$ & $3610 \mathrm{~B}$ & $5624 \mathrm{~A}$ & $28423 \mathrm{~A}$ & $13031 \mathrm{~A}$ & $2,19 \mathrm{~A}$ \\
$30 \mathrm{~cm}$ & $17097 \mathrm{AB}$ & $5776 \mathrm{~B}$ & $6387 \mathrm{~A}$ & $29260 \mathrm{~A}$ & $13440 \mathrm{~A}$ & $2,18 \mathrm{~A}$ \\
$40 \mathrm{~cm}$ & $13946 \mathrm{~B}$ & $7463 \mathrm{AB}$ & $6589 \mathrm{~A}$ & $27998 \mathrm{~A}$ & $13848 \mathrm{~A}$ & $2,06 \mathrm{~A}$ \\
$50 \mathrm{~cm}$ & $14425 \mathrm{~B}$ & $8728 \mathrm{~A}$ & $6239 \mathrm{~A}$ & $29392 \mathrm{~A}$ & $14175 \mathrm{~A}$ & $2,08 \mathrm{~A}$ \\
\hline C.V. $(\%)$ & 28,81 & 67,06 & 36,27 & 9,07 & 12,29 & 10,80 \\
\hline
\end{tabular}

${ }^{1} \mathrm{PNAC}$ - produção de frutos tipo mercado interno; PEXP - produção de frutos tipo exportação; PREF - produção de frutos refugo; PTOT - produção total de frutos; NTOT - número total de frutos; PMTOT - peso médio total de frutos. * Médias seguidas por letras maiúsculas iguais nas colunas não diferiram pelo teste de Tukey a $5 \%$ de probabilidade e letras minúsculas pelo teste $\mathrm{F}$ a $5 \%$ de probabilidade.

los fatores estudados, como também observaram Araújo (2000) e Ferreira (2001) estudando apenas a cobertura do solo. A profundidade de escarificação e a interação entre tipo de preparo e profundidade influenciaram significativamente a produção de frutos para o mercado interno (PNAC). O desdobramento da interação, apresentado na Tabela
3, mostra que não houve diferença pelo teste de Tukey a $5 \%$ de probabilidade entre as profundidades no preparo total, entretanto houve uma diminuição da PNAC à medida que aumentou a profundidade quando o preparo foi realizado em faixas. Este fato pode ser explicado pela concentração de água e nutrientes em um menor volume de solo quando o preparo é realizado em faixas e a pequenas profundidades, facilitando a exploração pelas raízes. Miranda et al. (2001), analisando os fatores de preparo do solo, obtiveram diminuição no consumo de combustível e tempo com a redução na largura da faixa preparada e na profundidade de escarificação. 
Apenas a profundidade de preparo influenciou a produção de frutos tipo exportação (PEXP), embora Araújo (2000) tenha observado menor produção do tipo exportação com o uso da cobertura com plástico. Na Tabela 2 se pode observar o aumento de PEXP com o aumento da profundidade de escarificação, comportamento inverso ao de PNAC. O coeficiente de variação elevado reflete a maneira subjetiva, e preferencial em relação aos outros tipos, como é feita a classificação do tipo exportação, que leva em conta menor tamanho de frutos, firmeza, coloração, formato e ausência de injúrias mecânicas. Pequenas profundidades de preparo parecem favorecer o acúmulo de umidade, o que foi comprovado por Araújo (2000) e Ferreira (2001), e de nutrientes como o nitrogênio, cujo excesso prejudica a qualidade e aparência dos frutos. As observações desses autores são reforçadas pelas tendências observadas, não significativas estatisticamente, de aumento de PNAC (14,85\%) e diminuição de PEXP (20,71\%) quando se usou cobertura plástica (Tabela 2), e pelos valores de PMTOT, cuja tendência foi apresentar frutos maiores no preparo em faixas, com cobertura e a menores profundidades.

A produção total de frutos de melão (PTOT) foi influenciada apenas pela interação entre tipo de preparo e cobertura do solo, cujo desdobramento mostra que quando se preparou o solo em área total o uso de cobertura do solo com plástico preto não influenciou significativamente a produção total $(29.384 \mathrm{~kg}$ $\mathrm{ha}^{-1}$ para sem cobertura e $28.483 \mathrm{~kg} \mathrm{ha}^{-1}$ para com cobertura). Mas, quando se preparou o solo em faixas, o uso da cobertura proporcionou uma produção total de $29.774 \mathrm{~kg} \mathrm{ha}^{-1}$, contra $27.443 \mathrm{~kg}$ $\mathrm{ha}^{-1}$ para sem cobertura. Esta diferença foi significativa pelo teste de Tukey a $5 \%$ de probabilidade. A cobertura do solo parece causar maior concentração de umidade e nutrientes próximo às raízes, o que beneficia o preparo em faixas se as raízes estiverem restritas em sua capacidade de obtê-los. Ferreira (2001) obteve maior produção de frutos comerciáveis quando usou cobertura, sem influência sobre a produção total. A ausência de constatação de efeito dos fatores individuais sobre a produ-

Tabela 3. Desdobramento da interação entre tipo de preparo e profundidade de preparo do solo para produção de frutos de melão tipo mercado interno $\left(\mathrm{kg} \mathrm{ha}^{-1}\right)$. Mossoró, ESAM, 2000.

\begin{tabular}{|c|c|c|}
\hline \multirow{2}{*}{ Profundidade } & \multicolumn{2}{|c|}{ Tipo de Preparo } \\
\hline & Total & Faixas \\
\hline 20 & $18.299 \mathrm{a}^{*}$ & 20.082 a \\
\hline 30 & 15.327 a & $18.868 a b$ \\
\hline 40 & 13.734 a & $14.157 \quad b c$ \\
\hline 50 & $17.250 \mathrm{a}$ & 11.601 \\
\hline
\end{tabular}

* Médias seguidas de letras iguais nas colunas não diferiram pelo teste de Tukey a 5\% de probabilidade.

ção total indica que o meloeiro se adaptou às condições adversas sem alterações significativas na sua capacidade de produção, mas com mudanças nas características de seus frutos.

Neste experimento, a cobertura do solo com plástico preto influenciou a qualidade dos frutos de melão causando redução nos valores de firmeza de polpa. A cobertura também proporcionou aumento da produção total de melão quando o preparo do solo foi realizado em faixas. Devido às implicações da cobertura do solo com o teor de água e de nutrientes no solo, deve-se proceder a novas pesquisas em relação ao manejo da irrigação e à fertilização. Quanto ao preparo do solo, o sistema de preparo em faixas não influenciou individualmente as características estudadas, podendo ser recomendado para a cultura do melão devido à economia de tempo e combustíveis proporcionada. Já, a profundidade de preparo exerceu influência sobre a produção de frutos tipo exportação, que aumentou com a profundidade, e sobre a produção para o mercado interno, que diminui com o aumento da profundidade no preparo em faixas. A produção total de melão não foi influenciada pelos fatores estudados, mas alguns de seus componentes responderam diferentemente a cada fator. Por exemplo, enquanto o tipo exportação aumentava com maiores profundidades o de mercado interno diminuía.

\section{LITERATURA CITADA}

ALLEN, R.G.; PEREIRA, L.S.; RAES, D.; SMITH, M. Crop evapotranspiration. Rome: FAO, 1998. 297 p.

ARAÚJO, A.P. Cobertura do solo e métodos de plantio no cultivo de melão amarelo. Mossoró: ESAM, 2000. 49 p. (Dissertação mestrado).
ARNDT, W.; ROSE, C.W. Traffic compaction and tillage requirements. Journal of Agricultural Engeneering Research, v. 11, n. 3, p. 170-187, 1966. FERREIRA, R.L.F. Produção e qualidade de melão cultivado sob condições climáticas resultantes de diferentes coberturas de solo e métodos de plantio. Mossoró: ESAM, 2001. 63 p. (Dissertação mestrado).

GRANGEIRO, L.C. Densidade de plantio em híbridos de melão amarelo. Mossoró: ESAM, 1997. 48 p. (Dissertação mestrado).

HILLEL, D. Introduction to soil physics. New York: Academic Press, 1982. 364 p.

HUNT, D. Farm power and machinery management. 9. ed. Ames: Iowa State University Press, 1995. 363 p.

IBARRA, L.; FLORES, J.; DIAZ-PÉREZ, J.C. Growth and yield of muskmelon in response to plastic mulch and row covers. Scientia Horticulturae, v. 87, n. 1-2, p. 139-145, 2001.

MAIERO, M.; SCHALES, F.D.; NG, J.T. Genotype and plastic mulch effects on earlines, fruit characteristics, and yield in muskmelon. Hortscience, v. 22, n. 5, p. 945-946, 1987.

McKIBBEN, J.S.; WHITAKER, F.D. Tilling various soil zones affects corn yields. Transactions of the ASAE, v. 16, n. 3, p. 404-406, 1973.

MIRANDA, N.O.; MEDEIROS, J.F.; ALVES, L.P.; MOURA NETO, E.L. Efeitos da profundidade do preparo do solo e do preparo em faixas sobre o desempenho operacional do trator e a produtividade do meloeiro (Cucumis melo l.). In: CONGRESSO BRASILEIRO DE ENGENHARIA AGRÍCOLA, 30, 2001, Foz do Iguaçú. Anais... Foz do Iguaçú: Sociedade Brasileira de Engenharia Agrícola/UNIOESTE, 2001. 1 CD-ROM.

OSCHWALD, W.R. Chisel plow and strip tillage systems. In: Conservation tillage. The proceedings of a national conference. Ames: Soil Conservation Society of America, 1973. p. 194-202.

SIEMENS, J.C.; HOEFT, R.G.; PAULI, A.W. Soil management. Moline: Deere \& Company Service Publications, 1993. 132 p.

SILVA, M.C.C. Crescimento, produtividade e qualidade de frutos de meloeiro sob diferentes níveis de salinidade da água de irrigação e cobertura do solo. Mossoró: ESAM, 2002. 65 p. (Dissertação mestrado).

TROUSE JR., A.C.; HUMBERT, R.P. Deep tillage in Hawaii: 1. Subsoiling. Soil Science, v. 88, n. 3, p. $150-159,1959$.

UNGER, P.W.; McCALLA, T.M. Conservation tillage systems. Advances in Agronomy, v. 33, p. 1-57, 1981. 